\title{
Exotic Earths: Forming Habitable Worlds with Giant Planet Migration
}

\author{
Sean N. Raymond, ${ }^{1,4,5 *}$ Avi M. Mandell, ${ }^{2,3,4,5 *}$ Steinn Sigurdsson ${ }^{2,4}$ \\ ${ }^{1}$ Laboratory for Atmospheric and Space Physics, University of Colorado \\ Boulder, CO 80309-0392 \\ ${ }^{2}$ Department of Astronomy and Astrophysics, Pennsylvania State University \\ University Park, PA 16802 \\ ${ }^{3}$ NASA Goddard Space Flight Center \\ Greenbelt, MD 20771 \\ ${ }^{4}$ Member of NASA Astrobiology Institute. \\ ${ }^{5}$ S.R. and A.M. contributed equally to this research. \\ *To whom correspondence should be addressed; E-mail: \\ raymond@lasp.colorado.edu, mandell@astro.psu.edu.
}

Close-in giant planets (e.g. "Hot Jupiters") are thought to form far from their host stars and migrate inward, through the terrestrial planet zone, via torques with a massive gaseous disk. Here we simulate terrestrial planet growth during and after giant planet migration. Several-Earth mass planets also form interior to the migrating Jovian planet, analogous to recently-discovered "Hot Earths". Very water-rich, Earth-mass planets form from surviving material outside the giant planet's orbit, often in the Habitable Zone and with low orbital eccentricities. More than a third of the known systems of giant planets may harbor Earth-like planets.

To date, giant planets have been detected around almost 200 main-sequence stars (1, 2). An 
unexpected result is the abundance of planets very close to their host stars - about $40 \%$ of the known extrasolar planets are interior to Mercury's orbital distance of 0.4 Astronomical Units (AU; $1 \mathrm{AU}$ is the Sun-Earth distance), although observational biases favor the detection of hot Jupiters (3). The occurence of close-in giant planets is surprising because models predict that giant planets form much more easily in the cold outer regions of protoplanetary disks (4) 5 ). These planetary systems have been attributed to inward migration of a giant planet on $10^{5}$ year timescales caused by an imbalance of torques generated by the gaseous protoplanetary disk (6 $6,8,8)$. In the process, the giant planet moves through the terrestrial planet zone (located from a few tenths of an AU to about 2-3 AU). Radioactive dating of Solar System material (10) and observations of dust dispersal in disks around young stars (11) indicate that rapid precipitation and coagulation of solid material in the inner regions of circumstellar disks is likely, leading to the question of the fate of these protoplanets during and after giant planet migration. Previous studies on the possibility of Earth-like planets co-existing with close-in giant planets are divided $(12,13,14,15 \mid 16)$.

Here we simulate the growth and dynamical evolution of protoplanetary material from small bodies to terrestrial planets during and after the migration of a giant planet through the terrestrial zone (see supporting online material for details). Simulations start from a circumstellar disk in the middle stages of planet formation, extending from 0.25 to $10 \mathrm{AU}$. The disk contains 17 Earth masses $\left(\mathrm{M}_{\oplus}\right)$ of rocky/icy material, evenly divided between 80 Moon- to Mars-sized "planetary embryos" (17) and 1200 "planetesimals" with properties modified so that each body behaves as collection of less massive objects (18). The disk has a compositional gradient: the inner disk is iron-rich and water-poor while the outer disk is water-rich and iron-poor (as in (19) but with 50\% water by mass beyond 5 AU.). A Jupiter-mass giant planet starts at $5 \mathrm{AU}$ and is migrated in to $0.25 \mathrm{AU}$ in $10^{5}$ years $(\underline{8})$. The orbits of all bodies in each simulation are integrated for 200 million years with the hybrid symplectic integrator Mercury (20), modified to include two 
additional effects: 1) "type 2" giant planet migration (13]6) and 2) aerodynamic gas drag (18) from a gaseous disk which dissipates on a $10^{7}$ year timescale (21).

At early times (Fig. 1) the giant planet migrates inward through the disk, causing nearby material to either be scattered outward onto high-eccentricity orbits (13) or shepherded inward by the giant planet's moving mean-motion resonances (22). The buildup of inner material induces rapid growth of a four Earth mass planet just inside the 2:1 mean motion resonance in $10^{5}$ years (also shown by (16)). Smaller bodies (planetesimals) feel a stronger drag force and are shepherded by higher-order resonances (in this case, the 8:1) and form a pileup of $0.2 \mathrm{M}_{\oplus}$ at $0.06 \mathrm{AU}$. At the end of the migration period, the remaining disk material is divided between bodies captured in low eccentricity orbits in interior resonances with the Jupiter-mass planet and higher eccentricity orbits beyond $0.5 \mathrm{AU}$. The protoplanetary disk is now dynamically hot (i.e. orbital eccentricities and inclinations are high), and accretion proceeds at a slower rate than would occur in a non-stirred, dynamically cold disk. However, the gas continues to damp eccentricities and inclinations, also causing the orbits of icy planetesimals from the outer disk to decay inwards on million year timescales, delivering a large amount of water to the growing terrestrial planets. After the gas dissipates (at $10^{7}$ years), the disk is stirred by interactions between bodies and clearing continues through scattering. After 200 million years the inner disk is comprised of the collection of planetesimals at $0.06 \mathrm{AU}$, a four Earth-mass planet at $0.12 \mathrm{AU}$, the Hot Jupiter at 0.21 AU, and a 3 Earth-mass planet at 0.91 AU. Previous results have shown that these planets are likely to be stable for billion year timescales (15). Many bodies remain in the outer disk, and accretion and ejection are ongoing due to long orbital timescales and high inclinations.

Two of the four simulations from Fig. 2 shown contain a $>0.3 \quad \mathrm{M}_{\oplus}$ planet on a loweccentricity orbit in the Habitable Zone, where the temperature is adequate for water to exist as liquid on a planet's surface (28). We adopt $0.3 \mathrm{M}_{\oplus}$ as a lower limit for habitability, includ- 
ing long-term climate stabilization via plate tectonics (23). The surviving planets can be broken down into three categories: 1) Hot Earth analogs interior to the giant planet, 2) "normal" terrestrial planets between the giant planet and 2.5 AU, and 3) outer planets beyond 2.5 AU, whose accretion has not completed by the end of the simulation. Properties of simulated planets are segregated (Table 1): Hot Earths have very low eccentricities and inclinations and high masses because they accrete on the migration timescale $\left(10^{5}\right.$ years $)$, so there is a large amount of damping during their formation. These planets are reminiscent of the recently discovered, close-in $7.5 \mathrm{M}_{\oplus}$ planet around GJ $876(\underline{24)}$, whose formation is also attributed to migrating resonances $(25)$. Farther from the star, accretion timescales are longer and the final phases take place after the dissipation of the gas disk (at $10^{7}$ years), causing the outer terrestrials to have large dynamical excitations and smaller masses, because accretion has not completed by 200 million years; collisions of outer bodies such as these may be responsible for dusty debris disks seen around intermediate-age stars (26). In the "normal" terrestrial zone, dynamical excitations and masses fall between the two extremes as planets form in a few times $10^{7}$ years, similar to the Earth's formation timescale (10). In addition, the average planet mass in the terrestrial zone is comparable to the Earth's mass, and orbital eccentricities are moderate (Table 1).

Both the Hot Earths and outer Earth-like planets have very high water contents (up to $>100$ times that of Earth (27)) and low iron contents compared with our own terrestrial planets (Table 1). There exist two sources for these trends in composition: 1) strong radial mixing induced by the migrating giant planet, and 2) an influx of icy planetesimals from beyond 5 AU from gas drag-driven orbital decay that is unimpeded by the scattering that Jupiter performs in our own system. The outer terrestrial planets acquire water from both of these processes, but the close-in giant planet prevents in-spiraling icy planetesimals from reaching the Hot Earths. The accretion of outer, water-rich material dilutes the high iron content of inner disk material, so water-rich bodies naturally tend to be iron-poor in terms of mass fraction. The high water contents of 
these planets suggest that their surfaces would be most likely covered by global oceans several $\mathrm{km}$ deep. Additionally, their low iron contents may have consequences for the evolution of atmospheric composition (29).

The spacing of planets (Fig. 2) is highly variable - in some cases planets form relatively close to the inner giant planet. The ratio of orbital periods of the innermost $>0.3 \mathrm{M}_{\oplus}$ terrestrial planet to the close-in giant ranges from 3.3 to 43 , with a mean[median] of 12[9]. We can therefore define a rough limit on the orbital distance of an inner giant planet that allows terrestrial planets to form in the Habitable Zone. For a terrestrial planet inside the outer edge of the Habitable Zone at 1.5 AU the giant planet's orbit must be inside roughly $0.5 \mathrm{AU}$ (the most optimistic case puts the giant planet at $0.68 \mathrm{AU})$. We apply this inner giant planet limit to the known sample of extra-solar giant planets (including planets discovered by the radial velocity, transit and microlensing techniques $(\underline{2,2)})$ in combination with a previous study of outer giant planets $(\underline{30})$. We find that 54 out of $158(34 \%)$ giant planetary systems in our sample permit an Earth-like planet of at least $0.3 \mathrm{M}_{\oplus}$ to form in the Habitable Zone (Fig. 3). The fraction of known systems that could be life-bearing may therefore be significantly higher than previous estimates (30).

The occurrence of Hot Jupiters appears to be a strong function of stellar metallicity (31). In addition, the solid component of protoplanetary disks is assumed to be proportional to metallicity. Therefore, systems such as the ones studied here may have very massive solid disks and could have systematically larger planet masses. If, for example, such disks are more likely to form $\sim 10 \mathrm{M}_{\oplus}$ "Hot Neptunes" (e.g., $55 \mathrm{Cnc}$ e (32)) than $\sim 4 \mathrm{M}_{\oplus}$ Hot Earths, then our disk is too small by a factor of a few. Assuming planet mass scales with disk mass, the typical mass of a habitable planet in such systems may be several Earth masses. In addition, our calculations were for a realistic but fixed giant planet mass and migration rate. Less[more] massive giant planets or faster[slower] migration rates increase[decrease] the survival rate of terrestrial 
material exterior to the close-in giant planet (13).

Upcoming space missions such as NASA's Kepler and Terrestrial Planet Finder and ESA's COROT and Darwin will discover and eventually characterize Earth-like planets around other stars. We predict that a significant fraction of systems with a close-in giant planet will be found to have a Hot Earth or potentially habitable, water-rich planets on stable orbits in the Habitable Zone. Suitable targets may be found in the known giant planet systems. 


\section{References and Notes}

1. Butler, R. P., et al. 2006, Astrophys. J., 646, 505

2. J. Schneider, The Extrasolar Planets Encyclopedia

(available at http://www.obspm.fr/encycl/encycl.html)

3. Tabachnik, S., \& Tremaine, S. 2002, Mon. Not. Roy. Ast. Soc., 335, 151

4. Bodenheimer, P., Hubickyj, O., \& Lissauer, J. J. 2000, Icarus, 143, 2

5. Boss, A. P. 1997 , Science, 276,1836

6. Lin, D. N. C., \& Papaloizou, J. 1986, Astrophys. J., 309, 846

7. Lin, D. N. C., Bodenheimer, P., \& Richardson, D. C. 1996, Nature, 380, 606

8. D’Angelo, G., Kley, W., \& Henning, T., 2003, Astrophys. J., 586, 540.

9. An alternative model for the presence of Hot Jupiters contends that they were dynamically scattered to small orbital distances, where tidal effects circularized their orbits (33). If that process is in fact dominant over the type 2 migration model considered here, then these models need to be re-considered. During the very high-eccentricity phase, it is likely that the giant planet could remove most of the terrestrial mass in the system, leaving little remaining to form planets.

10. Kleine, T., Münker, C., Mezger, K., \& Palme, H. 2002, Nature, 418, 952

11. Silverstone, M. D., et al. 2006, Astrophys. J., 639, 1138

12. Armitage, P. J., 2003. Astrophys. J., 582, L47.

13. Mandell, A. M. \& Sigurdsson, S. 2003. Astrophys. J., 591, L111. 
14. Edgar, R., \& Artymowicz, P. 2004, Mon. Not. Roy. Ast. Soc., 354, 769

15. Raymond, S. N., Quinn, T., \& Lunine, J. I. 2005. Icarus, 177, 256.

16. Fogg, M. J., \& Nelson, R. P. 2005, Astron \& Astrophys., 441, 791

17. Wetherill, G.W. \& Stewart, G.R. 1989, Icarus, 77, 330

18. Thommes, E. W., Duncan, M. J., \& Levison, H. F. 2003, Icarus, 161, 431

19. Raymond, S. N., Quinn, T., \& Lunine, J. I., 2004. Icarus, 168, 1.

20. Chambers, J. E., 1999. Mon. Not. Roy. Ast. Soc., 304, 793.

21. Haisch, K. E., Lada, E. A., \& Lada, C. J. 2001, Astrophys. J., 553, L153

22. Tanaka, H., \& Ida, S. 1999, Icarus, 139, 350

23. Williams, D. M., Kasting, J. F., \& Wade, R. A. 1997, Nature, 385, 234.

24. Rivera, E. J., et al. 2005, Astrophys. J., 634, 625

25. Zhou, J.-L., Aarseth, S. J., Lin, D. N. C., \& Nagasawa, M. 2005, Astrophys. J., 631, L85

26. Kim, J. S., et al. 2005, Astrophys. J., 632, 659

27. The Earth's total water content is between roughly 2 and 10 "oceans" (see (34)), where 1 ocean is the amount of water on our planet's surface $\left(1.5 \times 10^{24}\right.$ grams $)$; Earth is about $0.1 \%$ water by mass. Note also that although we did not account for water loss during impacts, water contents of these simulated planets are more than an order of magnitude higher than in previous simulations (19). So although the water contents listed in Table 1 are upper limits, the very high values are real.

28. Kasting, J. F., Whitmire, D. P., and Reynolds, R. T., 1993. Icarus 101, 108. 
29. Claire, M. Catling, D. \& Zahnle, K. 2006, Geobiology, in press.

30. Raymond, S. N. 2006, Astrophys. J., 643, L131

31. Fischer, D. A., \& Valenti, J. 2005, Astrophys. J., 622, 1102

32. McArthur, B. E., et al. 2004, Astrophys. J., 614, L81

33. Rasio, F. A., \& Ford, E. B. 1996, Science 274, 954

34. Lecuyer, C., Gillet, P., and Robert, F. 1998, Chemical Geology 145, 249.

35. Quinn, T. R., Tremaine, S., \& Duncan, M., 1991. Astronom. J., 101, 2287.

36. Hillenbrand, L. A., \& White, R. J. 2004, Astrophys. J., 604, 741

37. Lodders, K., \& Fegley, B. 1998, The planetary scientist's companion. Oxford University Press.

38. We thank NASA Astrobiology Institute for funding, through the Penn State (AM, SS), NASA Goddard (AM), Virtual Planetary Laboratory (SR) and University of Colorado (SR) lead teams. Thanks also to Jason Wright, Geoff Marcy and Paul Butler for early access to their new exoplanet orbits. Simulations were run on computers at Penn State and the University of Washington. 


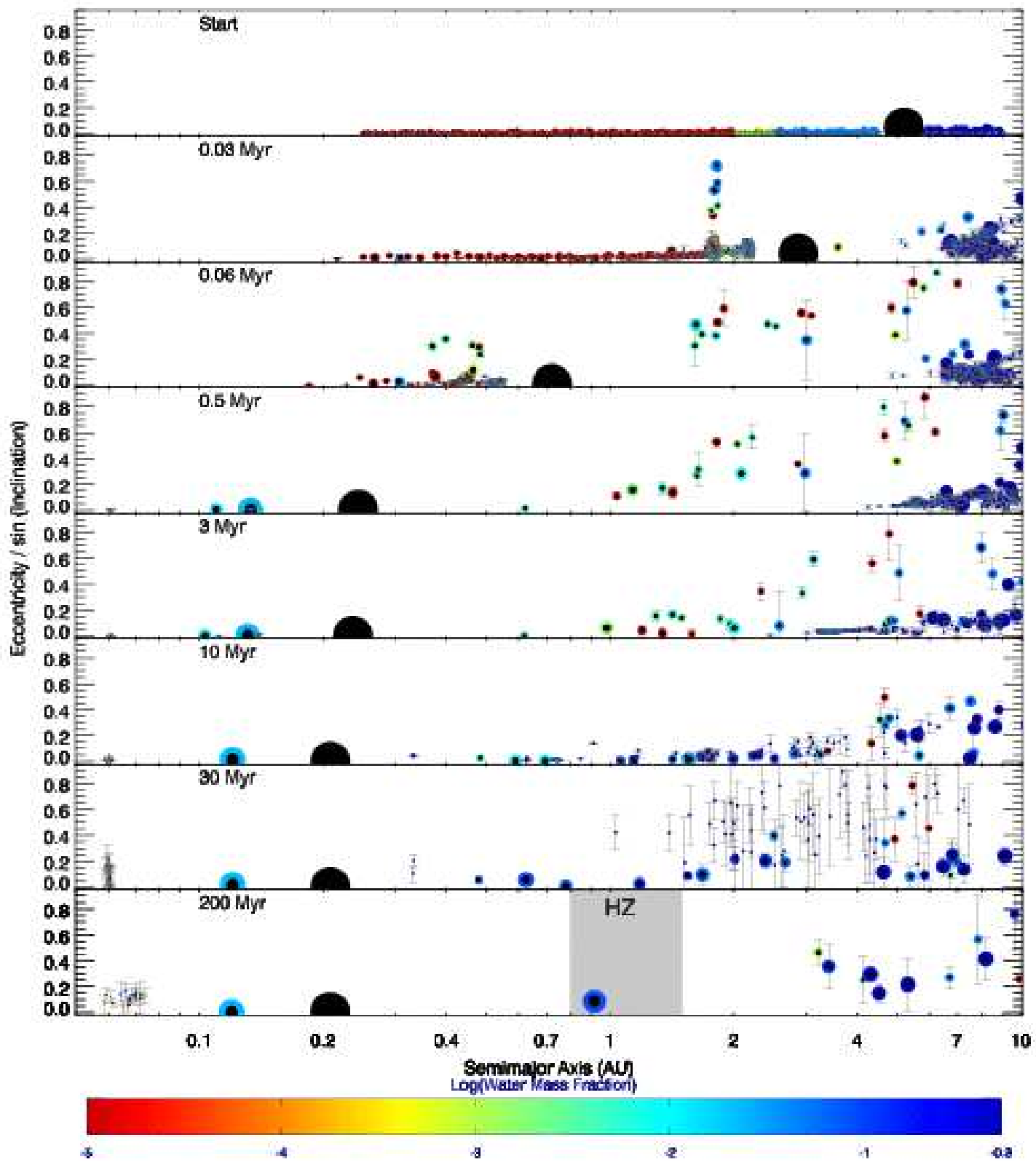

Figure 1 Snapshots in time of the evolution of one simulation. Each panel plots the orbital eccentricity vs. semimajor axis for each surviving body. The size of each body is proportional to its physical size (except for the giant planet, shown in black). The vertical "error bars" represent the Sine of each body's inclination on the y axis scale. The color of each dot corresponds to its water content (as per the color bar), and the dark inner dot represents the relative size of its iron core. For scale, the Earth's water content is roughly $10^{-3}$ (27). 


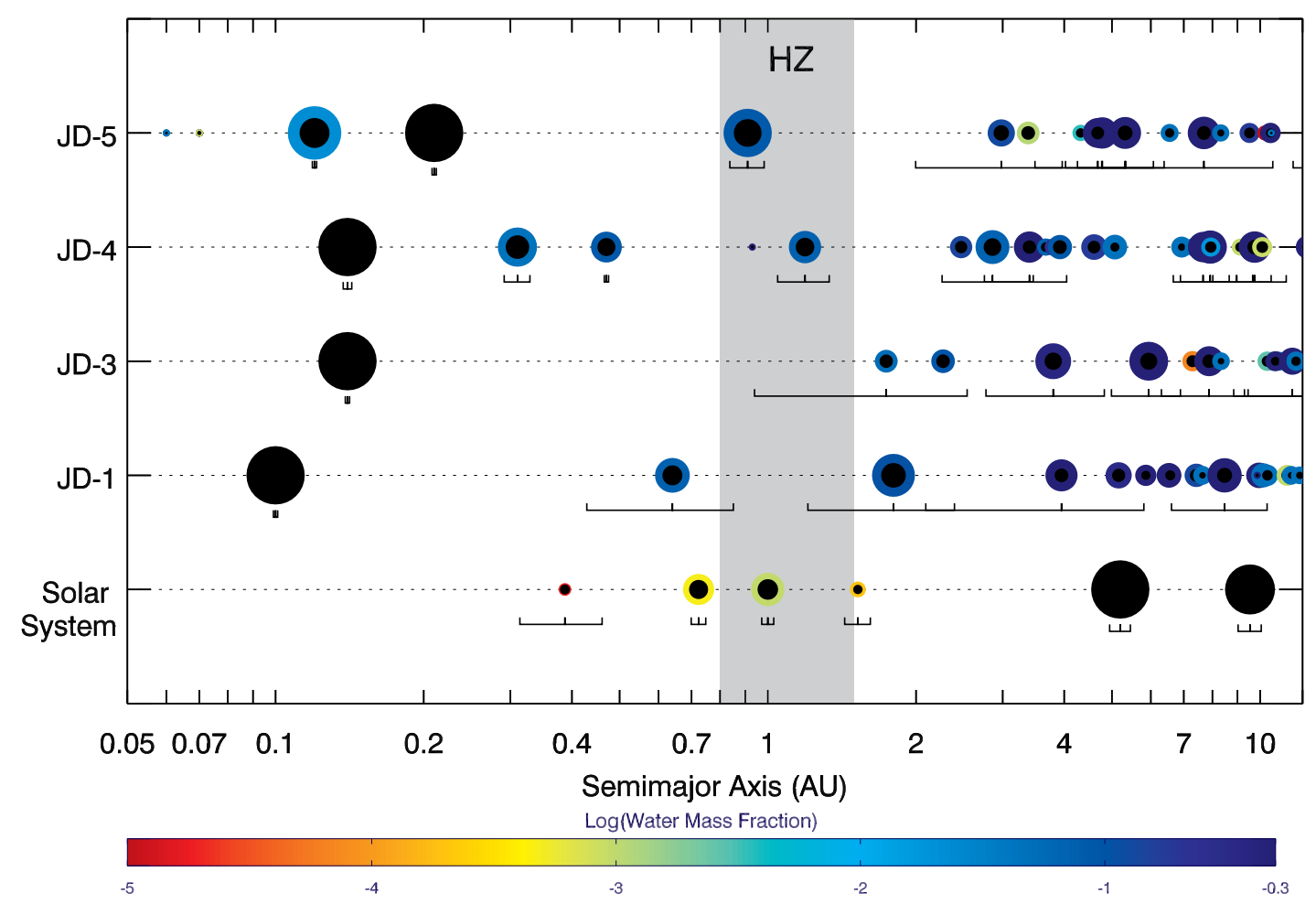

Figure 2 Final configuration of our four simulations, with the Solar System shown for scale. Each simulation is plotted on a horizontal line, and the size of each body represents its relative physical size (except for the giant planets, shown in black). The eccentricity of each body is shown beneath it, represented by its radial excursion over an orbit. As in Fig 1, the color of each body corresponds to its water content, and the inner dark region to the relative size of its iron core. The simulation from Fig. 1 is 'JD-5'. Orbital values are million year averages; Solar System values are 3 million year averages (35). See Table S1 for details of simulation outcomes. Note that some giant planets underwent additional inward migration after the end of the forced migration, caused by an articial drag force. This caused many Hot Earths to be numerically ejected, but had little effect outside the inner giant planet. See supporting online material for details. 


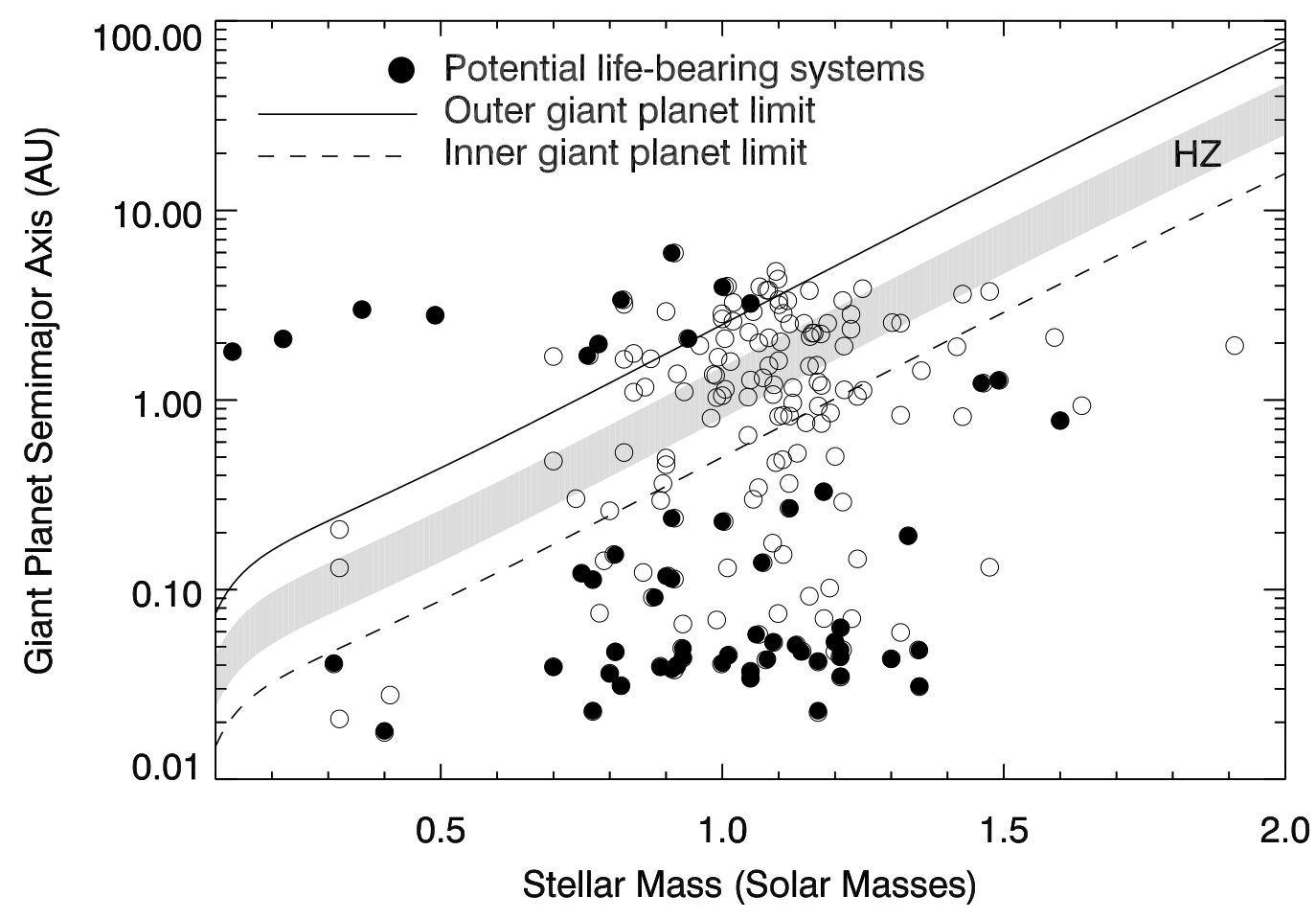

Figure 3 Giant planet orbital parameter space that allows terrestrial planets to form in the Habitable Zone. The solid line indicates the limit for outer giant planets from (30). The dashed line is an approximate limit $(0.5 \mathrm{AU}$ with eccentricity less than $0.1-$ the maximum eccentricity achieved in most simulations - for a solar-mass star) inside which low-eccentricity giant planets allow for the formation of habitable planets, derived from our results and (15). The Habitable Zone is shaded in green, and was calculated by assuming the temperature to scale with the stellar flux (i.e., the square root of the stellar luminosity), using a stellar mass-luminosity relation fit to data of (36). Open circles represent known giant planets that are unlikely to allow habitable terrestrial planets in the Habitable Zone. Filled circles represent known planets with low enough orbital eccentricities to satisfy our criteria for habitable planet formation, deemed to be potentially life-bearing. 
Table 1. Properties of Simulated Planets ${ }^{1}$

\begin{tabular}{l|ccccc}
\hline \hline & Hot Earths & Normal Terrestrials & Hab. Zone planets & Outer Terrestrials $^{\text {Solar System }}{ }^{2}$ \\
\hline Mean Number of planets & $0.25^{3}$ & 2 & 0.5 & 11 & 0.4 \\
Mean Planet mass ( $\left.M_{\oplus}\right)$ & $4.2^{-2}$ & 1.1 & 2.0 & 0.6 & 0.49 \\
Mean Water mass fraction & $2 \times 10^{-2}$ & $8 \times 10^{-2}$ & $8 \times 10^{-2}$ & $3.5 \times 10^{-1}$ & $4 \times 10^{-4}$ \\
Mean Iron mass fraction & 0.25 & 0.28 & 0.27 & 0.14 & $0.32^{4}$ \\
Mean Orbital eccentricity & 0.01 & 0.23 & 0.10 & 0.23 \\
Mean Orbital inclination (deg) & 0.7 & 11 & 7 & 13 \\
\hline
\end{tabular}

${ }^{1}$ We include results from four simulations, described in detail in the supporting online material.

${ }^{2}$ Solar System physical properties from 37 and orbital properties from 35 ).

${ }^{3}$ Every simulation with gas drag formed 1-3 Hot Earths during giant planet migration. However, in many cases an artificial drag force caused continued inward migration of the giant planet. In these cases, the integrator usually introduced an error causing the eventual ejection of Hot Earths when they entered $\sim 0.05 \mathrm{AU}$. We therefore consider 0.5 a lower bound on the frequency of Hot Earths in these systems. See also (16).

${ }^{4}$ Solar System iron mass fraction are calculated without Mercury, because of its anomalously high iron content. 